\title{
Contextual strength does not modulate the subordinate bias effect: Evidence from eye fixations and self-paced reading
}

\author{
KATHERINE S. BINDER and KEITH RAYNER \\ University of Massachusetts, Amherst, Massachusetts
}

\begin{abstract}
Eye movements were recorded in order to examine how different sources of information-namely, meaning dominance and strength of biasing context-influence the processing of biased ambiguous words. Gaze durations were longer on ambiguous target words when the preceding context instantiated the subordinate interpretation, even with strongly biasing contexts. Identical results were obtained with a self-paced reading study. Thus, contrary to recent findings (Kellas, Martin, Yehling, Herman, \& Vu, 1995), the subordinate interpretation of a biased ambiguous word was not selectively accessed even when the preceding context strongly biased that interpretation. Discrepancies between the present experiments and the Kellas et al. experiment are discussed.
\end{abstract}

A considerable amount of research has been devoted to examining how different sources of constraint influence the processing of lexically ambiguous words. From this research, it is clear that contextual information and relative meaning frequency both impact lexical ambiguity resolution (Binder \& Morris, 1995; Dopkins, Morris, \& Rayner, 1992; Duffy, Morris, \& Rayner, 1988; Neill, 1989; Neill, Hilliard, \& Cooper, 1988; Rayner, Pacht, \& Duffy, 1994; Simpson, 1984). Exactly how these sources of information combine to influence the resolution process has been a greatly debated question. At issue is whether the language processing system can be viewed as highly interactive (see, e.g., Marslen-Wilson, 1975) or modular in nature (Forster, 1979). Lexical ambiguity has proven to be a useful tool in understanding the nature of the language processing system in that activation of only the contextually appropriate meaning (selective access) provides evidence for an interactive view, while activation of both meanings (exhaustive access), in spite of biasing context, suggests a modular system.

A number of recent eye movement studies have examined how lexically ambiguous words are processed (Binder \& Morris, 1995; Dopkins et al., 1992; Duffy et al., 1988; Rayner \& Duffy, 1986; Rayner \& Frazier, 1989; Rayner et al., 1994; Sereno, 1995; Sereno, Pacht, \& Rayner, 1992). In these studies, readers' eye movements were monitored as they read sentences or short paragraphs, and fixation

This research was supported by Grant HD 17246 from the National Institutes of Health and was completed when K.S.B. was a postdoctoral fellow on Grant MH16745 from the National Institute of Mental Health. K.R. was supported by a Research Scientist Award (MH01255). We thank George Kellas and Charles Martin for providing the stimulus materials. We also thank Derek Besner, Curt Burgess, George Kellas, Randi Martin, Trammell Neill, and Hoang Vu for their comments on a prior version of this article. Correspondence should be addressed to K. Rayner, Department of Psychology, University of Massachusetts, Amherst, MA 01003 (e-mail: rayner@psych.umass.edu). time on an ambiguous word or a control word (that was matched in length and frequency) was measured. A consistent pattern of results has emerged from these studies. When neutral context precedes the ambiguous word, readers fixate longer on balanced ambiguous words (words that have two equally likely meanings) than on biased words (words that have one dominant interpretation) or on an unambiguous control word. However, readers spend significantly more time in the disambiguating region following the target word in the case of biased words that are disambiguated toward the subordinate interpretation. When disambiguating information precedes the ambiguous word, the pattern of results is quite different. Fixation times are longer on the biased ambiguous words when the preceding context favors the subordinate meaning than on either the balanced words or the unambiguous control words. Rayner et al. referred to this latter finding as the subordinate bias effect and found that it is obtained even when subjects read passages in which the subordinate meaning of the ambiguous word was repeated across the passage, and the global context of the passage was biased toward the subordinate interpretation. Thus, Rayner et al. concluded that the subordinate interpretation of a biased ambiguous word was not selectively accessed.

The best account of the overall pattern of data is provided by the reordered access model (Duffy et al., 1988). According to this model, the resting activation of the two meanings is roughly equivalent in the case of balanced ambiguous words. The context that precedes the ambiguous word works to boost the activation of the contextually relevant meaning so that it is accessed prior to the contextually inappropriate meaning. As a result, lexical access time is not increased because no subsequent selection process is required. The contextually appropriate meaning is integrated with the prior context in the same manner as the unambiguous control word. In the case of biased ambiguous words, however, the preceding biasing context 
boosts the activation of the subordinate meaning so that it is accessed at or near the same time as the dominant interpretation. The resulting inflated processing times on these words are due to a time-consuming selection process between the meanings. Thus, this account suggests that access of lexically ambiguous words is exhaustive, but not autonomous, in that factors such as relative meaning frequency and contextual bias influence the order in which the words are accessed.

Another model that also takes into consideration meaning frequency and contextual bias is the context-sensitive model proposed by Kellas and colleagues (Kellas, Martin, Yehling, Herman, \& Vu, 1995; Kellas, Paul, Martin, \& Simpson, 1991; Kellas, Vu, Martin, \& Herman, 1994; Paul, Kellas, Martin, \& Clark, 1992). In contrast to the reordered access model, selective access of a biased ambiguous word can occur because context that precedes the ambiguous word can activate either one meaning or both meanings depending on the strength of the context. That is, when the context is weak, relative meaning frequency will have a greater influence in the meaning activation process. However, when the prior context is strongly biased toward one interpretation, only the contextually appropriate meaning will be activated regardless of meaning frequency. In contrast to the above-mentioned eye movement studies, experiments that have been used to support this position do not demonstrate the subordinate bias effect when the context that precedes the ambiguous word is strongly biased; rather, the subordinate bias effect has been obtained only when the context has been weakly biased toward the subordinate interpretation (Kellas et al., 1995; Kellas et al., 1991; Paul et al., 1992).

Kellas et al. (1995) argued that the discrepancy between their results and those obtained by Rayner et al. (1994) was due to the lack of strongly biasing contextual information in the Rayner et al. passages. However, another alternative for why some studies obtained evidence of selective access of the subordinate meaning is task differences (in Kellas's experiments, self-paced reading, naming, and Stroop tasks have all been used). That is, some tasks may not be sensitive enough to detect multiple access. In order to directly examine these explanations, we obtained copies of the passages that were used in the Kellas et al. (1995) study and carried out two experiments using these passages. In these experiments, the influence of two different sources of information (meaning dominance and strength of preceding context) on word processing was examined. In Experiment 1, subjects' eye movements were monitored as they read two sentence passages, which created a bias consistent with either the dominant or the subordinate meaning of the ambiguous word. In Experiment 2, the self-paced reading procedure employed by Kellas et al. (1995) was used. The biasing context was the first sentence of the passage, and it (1) established a character who was performing an activity or (2) described an attribute of the character. The context of the initial sentence established either a strong or a weak bias for the intended meaning. The second sentence al- ways began with a pronoun that referred to the character introduced in the initial sentence followed by a verb and then the ambiguous word. We created control conditions by replacing the ambiguous target word with an unambiguous word that was matched on length and frequency (see Table 1 for example passages). The predictions that follow from the reordered access model and the contextsensitive model are quite straightforward. According to the reordered access model, the subordinate bias effect will be obtained even when the preceding context is strongly biased. According to the context-sensitive model, the subordinate bias effect will be obtained when the context that precedes the ambiguous word weakly biases the subordinate meaning, but the effect will not surface when the context is strongly biased.

\section{EXPERIMENT 1}

\section{Method}

Subjects. Twenty-eight students at the University of Massachusetts participated in the study. They either received class credit or were paid $\$ 8$ for participating. All subjects had uncorrected vision and were native English speakers.

Procedure. Each subject read a series of short passages presented on a computer screen. Subjects were told to read for comprehension and that from time to time they would be asked a question about the passage they just read, which could be answered by responding "yes" or "no." In addition, it was stressed that they should read normally, including rereading if necessary. After each subject understood the procedure and informed consent was obtained, a bite bar was prepared to minimize head movement. The eyetracking system was then calibrated to the subject. This procedure took approximately $5 \mathrm{~min}$ for each subject.

At the beginning of each trial, five boxes appeared on the screen and the subject was instructed to look at the left-most box. Once the experimenter had determined that the subject was fixating the box, the passage was presented on the screen to begin the trial. When the subject was finished reading the passage, he/she pushed a button, ending that trial. Subjects were asked comprehension questions on approximately $25 \%$ of the passages (they answered correctly $97 \%$ of the time). No individual items were excluded from the analyses on the basis of answers to comprehension questions.

Apparatus. Eye movements were recorded by a Fourward Technologies Dual Purkinje Image eyetracker. Although viewing was binocular, only the right eye was monitored. The eyetracker has a resolution of $10^{\prime}$ of arc. The system was interfaced with an Epson Equity III computer. The passages were presented in a double-spaced format on a Sony monitor. The subject was seated $80 \mathrm{~cm}$ from the monitor, with four letters of text subtending $1^{\circ}$ of visual angle. The brightness of the screen was adjusted for each subject to ensure comfort. The passages were two lines long, with approximately 60 characters per line.

Table 1 Example Passage From the Experiment

\begin{tabular}{|c|c|}
\hline Weak Context & Strong Context \\
\hline \multicolumn{2}{|c|}{ Dominant } \\
\hline $\begin{array}{l}\text { The agent revealed the strategy. } \\
\text { He described the bluff (offer) } \\
\text { to the other officers. }\end{array}$ & $\begin{array}{l}\text { The robber rushed out the door. } \\
\text { He came from the bank (shop) with } \\
\text { both guns blazing. }\end{array}$ \\
\hline \multicolumn{2}{|c|}{ Subordinate } \\
\hline $\begin{array}{l}\text { The scout returned home. } \\
\text { He described the bluff (cliff) } \\
\text { to the little children. }\end{array}$ & $\begin{array}{l}\text { The alligator saw the food. } \\
\text { He came from the bank (edge) with } \\
\text { a quick lunge. }\end{array}$ \\
\hline
\end{tabular}

Note-The target word is italicized, and the unambiguous control word is in parentheses. 
Materials. Thirty-two ambiguous words ${ }^{1}$ were chosen for Experiment 1. Each of these words was embedded into two paragraph framesone that biased the dominant sense of the ambiguous word and one that biased the subordinate interpretation. Half of the ambiguous words were preceded by strongly biasing context, while the remaining words were preceded by weakly biasing context. ${ }^{2}$ In addition, control conditions were established by replacing the ambiguous target word with a word matched on length and frequency according to the Francis and Kučera (1982) norms. The average word frequency for the ambiguous words was 59 , while the mean for the control words was 57 . Thus, three factors were manipulated: meaning biased by the context (dominant or subordinate), strength of context (strong or weak), and word type (ambiguous or control). All manipulations were made within subjects, using a Latin square design. In addition to the 32 experimental passages, 48 filler passages were used. The passages were presented to each subject in a different random order.

\section{Results and Discussion}

Gaze duration on the target word, or the sum of all consecutive fixations on a word before leaving that word (not including any regressions), was measured. ${ }^{3}$ If the target word was not fixated, the closest fixation within three letter spaces to the left and one letter space to the right of the target was counted as the fixation during which the target word was processed (Rayner \& Pollatsek, 1989). In addition, the first fixation following the target word was examined as a measure of spillover processing time (Rayner \& Duffy, 1986). Fixations under $120 \mathrm{msec}$ were eliminated from the analysis because such short fixations are thought to reflect oculomotor programming (Morrison, 1984). Fixations longer than $800 \mathrm{msec}$ were assumed to be the result of momentary track losses or eye blinks and were also eliminated. Altogether, $7 \%$ of the data were eliminated from the analyses.

Gaze duration on the target word. More time was spent on ambiguous target words when the preceding context instantiated the subordinate meaning than in the other conditions (Table 2). Subjects fixated on the ambiguous target word for $299 \mathrm{msec}$ when the subordinate meaning was instantiated and for $276 \mathrm{msec}$ when the dominant meaning was instantiated by the context. Gaze durations on the control words were 271 and $278 \mathrm{msec}$ for the subordinate and dominant conditions, respectively. This pattern was maintained regardless of contextual strength. This was demonstrated by a significant interaction of dominance and word type $\left[F_{1}(1,27)=6.8\right.$, $M S_{\mathrm{e}}=1,824, p<.01$, and $F_{2}(1,31)=5.3, M S_{\mathrm{e}}=2,090$, $p<.04]$. Importantly, the three-way interaction, which was predicted by the context-sensitive model, was not significant $(F \mathrm{~s}<1)$. Post hoc tests revealed that gaze durations on the ambiguous subordinate target word differed from the gaze durations in the other three conditions $(p s<.05)$, which did not differ from one another $(t \mathrm{~s}<1)$.

There are two ways to assess the subordinate bias effect. One way, which has typically been used in eye movement experiments, is to compare the gaze duration on the ambiguous target word with its appropriate control word. For the strong-context condition, this yielded a $33-\mathrm{msec}$ effect $(p<.01)$, while for the weak-context condition the effect was $24 \mathrm{msec}(p<.05)$. A second way, which was used by Kellas et al. (1995), is to compare when the dom-
Table 2

Processing Time for the Target Word and Spillover (in Milliseconds)

\begin{tabular}{lccccc}
\hline & \multicolumn{2}{c}{ Target Word } & & \multicolumn{2}{c}{ Spillover } \\
\cline { 2 - 3 } Context & Dominant & Subordinate & & Dominant & Subordinate \\
\hline Weak & \multicolumn{5}{c}{ Experiment 1} \\
$\quad$ Target & 281 & 294 & & 250 & 281 \\
$\quad$ Control & 281 & 270 & & 252 & 254 \\
Strong & & & & \\
$\quad$ Target & 271 & 304 & & 265 & 269 \\
Control & 274 & 271 & & 266 & 265 \\
& & Experiment 1A & &
\end{tabular}

\begin{tabular}{|c|c|c|c|c|}
\hline \multicolumn{5}{|l|}{ Weak } \\
\hline Target & $300(316)$ & $325(304)$ & $260(263)$ & $267(281)$ \\
\hline Control & $303(287)$ & $302(275)$ & $256(262)$ & $262(254)$ \\
\hline \multicolumn{5}{|l|}{ Strong } \\
\hline Target & $277(300)$ & $270(283)$ & $252(265)$ & $266(269)$ \\
\hline \multirow[t]{2}{*}{ Control } & $269(288)$ & $278(262)$ & $262(266)$ & $255(250)$ \\
\hline & & \multicolumn{3}{|c|}{ Experiment 2} \\
\hline \multicolumn{5}{|l|}{ Weak } \\
\hline Target & 553 & 571 & 512 & 552 \\
\hline Control & 554 & 556 & 496 & 512 \\
\hline \multicolumn{5}{|l|}{ Strong } \\
\hline Target & 510 & 556 & 499 & 498 \\
\hline Control & 503 & 517 & 501 & 508 \\
\hline
\end{tabular}

Note-For Experiments 1 and $1 \mathrm{~A}$, the time on the target word is the gaze duration and the spillover time is the duration of the next fixation. For Experiment 2, the spillover time is for the next word in the text. The values in parentheses for Experiment $1 \mathrm{~A}$ represent the mean gaze durations once the problematic items were removed from the full set of materials provided by Kellas et al. (1995).

inant meaning of the ambiguous word is instantiated with when the subordinate meaning is instantiated. This comparison yielded a 34-msec subordinate bias effect $(p<.01)$ for the strong context and a marginally significant $13-\mathrm{msec}$ effect for the weak context $(p<.10)$.

First fixation following the target word. More time was spent on the first fixation following the ambiguous target word when the preceding context weakly supported the subordinate interpretation (Table 2). This was borne out in the three-way interaction, which was significant by subjects $\left[F_{1}(1,27)=4.1, M S_{\mathrm{e}}=1,217, p<\right.$ $.05]$ and marginally significant by items $\left[F_{2}(1,31)=3.04\right.$, $\left.M S_{\mathrm{e}}=1,970, p<.07\right]$. A two-way analysis of variance (ANOVA) for word type (target vs. control) and meaning dominance (dominant vs. subordinate) when the context was weakly biasing revealed that spillover fixations for the ambiguous subordinate target word differed from the fixations in the other three conditions $\left[F_{1}(1,27)=4.84\right.$, $M S_{\mathrm{e}}=2,445, p<.05 ; F_{2}(1,15)=5.31, M S_{\mathrm{e}}=1,950$, $p<.05]$. There were no significant differences among the conditions when the preceding contextual strength was strong (all $F_{\mathrm{S}}<1$ ). Thus, strength of context had a clear effect on the first fixation following the target word. For the strong context, there was no difference between the ambiguous word and its control when the subordinate meaning was instantiated (nor was there a dif- 
ference between the dominant and the subordinate meanings). However, the pattern was quite different for the weak context. Specifically, the subordinate meaning yielded fixations that were $27 \mathrm{msec}$ longer than those for the control word $(p<.01)$, and the subordinate meaning yielded fixations that were $31 \mathrm{msec}$ longer than those for the dominant meaning $(p<.01)$.

The results of Experiment 1, then, with respect to the target word, are consistent with results reported in previous eye movement experiments and support the reordered access model. However, the question remains why we obtained a different pattern of data from that of Kellas and his colleagues (1995). As mentioned in the introduction, the discrepancy between the pattern associated with the two positions has been attributed to differences in the task, the materials, or a combination of these factors. In Experiment 2, we specifically examined the extent to which task differences may account for the difference, but here we will focus on the materials.

Initially, we ran the entire materials set from the Kellas et al. (1995) study. Using their full set of materials, we recorded the eye movements of 24 subjects and obtained a pattern very similar to the one they reported (see Experiment $1 \mathrm{~A}$ in Table 2). However, after the study was run, a careful analysis of the items used by Kellas et al. (1995) revealed three potential concerns. First, according to our local norms, as well as the Twilley, Dixon, Taylor, and Clark (1994) norms, 14 of the ambiguous words used by Kellas et al. (1995) were balanced ambiguous words. Previous eye movement experiments have demonstrated that when context precedes a balanced ambiguous word, processing time on the ambiguous word is not different from that on an unambiguous control word (Binder \& Morris, 1995; Duffy et al., 1988; Rayner \& Frazier, 1989). That is, the context works to boost the activation of the contextually appropriate meaning so that it is accessed prior to the inappropriate meaning. Thus, processing differences (and hence gaze duration differences) are not expected between the ambiguous words and control words. Second, in seven of the items, again according to local norms and the Twilley et al. norms, the bias information was reversed (contexts biasing the dominant meaning were really biasing the subordinate meaning). Third, there were three items in which the different contexts (dominant biasing and subordinate biasing) actually biased the same meaning. To illustrate this point, consider the following example of the ambiguous word date. The dominant biasing context was, "The student made an error. He forgot the date on the final exam." The subordinate biasing context was, "The boyfriend was thoughtless. He forgot the date of the birthday party." Both contextual forms refer to the dominant meaning of date, while the subordinate meaning (fruit) is absent from either context. Frazier and Rayner (1990) demonstrated that words with multiple senses (as in the date example) yielded a different pattern of reading times than did truly lexically ambiguous words.
Given these concerns with the full materials set, we did two things. First, we eliminated the problematic items from the data set; the resulting means (see the values in parentheses in Table 2) were quite consistent (when the ambiguous target words are compared with the control words) with the data we obtained in Experiment 1 . Second, and more importantly, because there was random variability due to the lack of counterbalancing in the corrected means from Experiment $1 \mathrm{~A}$, we selected the remaining items and ran the experiment reported earlier. The results of the eye movement experiments led us to suspect that the difference between the pattern of results was due to the materials used. However, the possibility remained that task differences might account for the differences. In particular, since self-paced reading yields longer times per word, and since we did obtain a pattern like Kellas et al. (1995) obtained in self-paced reading on the spillover fixation, it might be that the slower processing characteristic of self-paced reading would yield the pattern of data obtained by Kellas et al. for the refined set of materials. Experiment 2 was carried out to examine this possibility.

\section{EXPERIMENT 2}

\section{Method}

Subjects. Thirty-six students at the University of Massachusetts participated in the study. They received class credit for participating.

Procedure and Materials. Each subject read the passages from Experiment 1 on a computer screen. A word-by-word self-paced reading procedure was used in which subjects advanced through the text by pushing a button to display the next word in the text. The locations of to-be-read words were indicated by dashes. Except for the difference in the method used to assess reading times for the target words, all other aspects of the study were the same as in Experiment 1. Subjects answered the comprehension questions correctly $98 \%$ of the time.

\section{Results and Discussion}

Reading time on the target word was measured. In addition, the time on the word that followed the target word was examined as a measure of spillover. As seen in Table 2, the pattern of results from Experiment 2 were nearly identical to that of Experiment 1.

Reading time on the target word. More time was spent reading the ambiguous target word when the preceding context instantiated the subordinate meaning than in the other conditions. Readers looked at the ambiguous target word for $563 \mathrm{msec}$ when the subordinate meaning was instantiated and for $532 \mathrm{msec}$ when the dominant meaning was instantiated by the context. Reading times for the control words were 537 and $529 \mathrm{msec}$ for the subordinate and dominant conditions, respectively. As in Experiment 1, this pattern was maintained regardless of contextual strength, as demonstrated by a significant interaction of dominance and word type $\left[F_{1}(1,35)=7.3\right.$, $M S_{\mathrm{e}}=3,268, p<.05$, and $F_{2}(1,31)=6.7, M S_{\mathrm{e}}=5,897$, $p<.05]$. Once again, the three-way interaction predicted by the context-sensitive model was not significant $(F \mathrm{~s}<$ 1). Post hoc tests revealed that reading time for the am- 
biguous subordinate target word differed $(p<.05)$ from reading times in the other three conditions, which did not differ from one another.

Using the two methods described earlier to assess the subordinate bias effect, we again found that the effect is larger with the strong context than with the weak context. Specifically, when responses to the ambiguous target word were compared with those to its control word, the subordinate bias effect was $39 \mathrm{msec}(p<.01)$ for the strong context and $15 \mathrm{msec}(p<.08)$ for the weak context. When the two meanings of the ambiguous word were compared, the subordinate bias effect was $46 \mathrm{msec}(p<.01)$ for the strong context and $18 \mathrm{msec}(p<.07)$ for the weak context.

Reading time for the next word. More time was spent reading the word following the ambiguous target word when the context weakly supported the subordinate meaning (Table 2). This was borne out in the three-way interaction $\left[F_{1}(1,35)=5.6, M S_{\mathrm{e}}=3,268, p<.05\right.$, and $\left.F_{2}(1,31)=4.56, M S_{\mathrm{e}}=5,687, p<.05\right]$. A two-way ANOVA for word type (target vs. control) and meaning dominance (dominant vs. subordinate) when the context was weakly biasing revealed that reading time for the ambiguous subordinate target word differed from the reading times in the other three conditions $\left[F_{1}(1,35)=\right.$ $5.3, M S_{\mathrm{e}}=2,357, p<.05 ; F_{2}(1,15)=4.9, M S_{\mathrm{e}}=3,269$, $p<.05]$. There were no significant differences among the conditions when the preceding contextual strength was strong (all $F \mathrm{~s}<1$ ).

\section{GENERAL DISCUSSION}

The purpose of the present experiments was to examine how sources of information, namely meaning dominance of biased ambiguous words and strength of biasing context, influence the processing of ambiguous words. The results of the experiments were quite straightforward. More processing time was spent on the ambiguous target words when the preceding context instantiated the subordinate sense (the subordinate bias effect) than on the unambiguous control words or on the ambiguous target words when the context biased the dominant interpretation. Although this pattern was maintained, statistically speaking, regardless of contextual strength, careful examination of the data revealed that the subordinate bias effect was stronger for the strong context than for the weak context. That is, the subordinate bias effect was on the order of 33-46 msec for the strong-context condition (with the effect computed either by subtracting the time spent processing the control word from that spent processing the ambiguous word or by subtracting the times for the dominant condition from those for the subordinate condition) across the two main experiments reported here. By contrast, the subordinate bias effect was on the order of 13-24 msec for the weak context. This pattern was obtained in both experiments (eye movements as well as the self-paced reading experiment). This pattern of results is quite consistent with the reordered access model, since the stronger contex would serve to more readily boost the subordinate interpretation of the ambiguous word than would the weaker biasing context. Strength of context clearly influenced the spillover measures examined here. When the context weakly biased the subordinate meaning of the ambiguous word, more time was spent on the first fixation following the target word in Experiment 1 and on the word following the ambiguous target word in Experiment 2 compared with all other conditions.

A selective access model has difficulty accounting for the subordinate bias effect. If context aids the selection of the appropriate sense, it is unclear why the selection process would take longer for the biased words. Thus, the pattern of data for both experiments reported here for the target word is inconsistent with the context-sensitive model of lexical ambiguity processing (Kellas et al., 1995). We also note at this point that in addition to collecting the norming data on the strength-of-context manipulation (see note 2), we also collected similar norms on the strength of the biasing contexts used by Duffy et al. (1988). In two separate norming studies, subjects were told that they would be reading short sentences (Duffy et al. norming task) or passages (Kellas et al., 1995, norming task) that contained a word that had more than one meaning. Prior to each sentence or passage, the ambiguous word was presented along with a short definition of the meaning that was intended by the sentence or passage. This was followed by the presentation of the sentence or passage up to and including the ambiguous target word. The subject was asked to rate how well the context biased the provided meaning of the ambiguous word. Interestingly, we found that Duffy et al.'s contexts were rated as stronger than the strong contexts of Kellas et al. (1995): On a 7-point scale $(1=$ weakly biasing; $7=$ strongly biasing), the Kellas et al. contexts received a rating of 5.1, while the Duffy et al. contexts received a rating of 5.9. This fact, together with the results of the experiments reported here, clearly indicate that strong biasing context does not eliminate the subordinate bias effect. We suspect that Kellas et al. obtained a different pattern of results due to the materials they used. Of course, we do not know whether the Nelson et al. (1990) norms are appropriate for Kellas et al.'s (1995) subjects. However, the local norms we have collected seem more consistent with the Twilley et al. (1994) norms than with the Nelson et al. norms. Given that there are obviously geographical differences in the norms, perhaps the most appropriate procedure is to collect local norms.

Although the pattern of data on the target word was not consistent with the context-sensitive model, the spillover data were consistent with the predictions from this model. When the preceding context weakly biased the subordinate interpretation, inflated spillover processing times following the target word were associated with the ambiguous target compared with those in the other conditions. There are two plausible explanations for this effect. First, it is possible that although strong context did not produce selective access on the target word itself, it may have aided in the resolution process so that processing difficulty did not continue past the ambiguous target word. The second alternative is simply that initially, the weak context did not provide enough support for the subordinate interpretation. Thus, the conflict between the two meanings was delayed until the word following the target.

Overall, the pattern of data was not consistent with the contextsensitive view of lexical ambiguity processing (Paul et al., 1992). The data are consistent, however, with the reordered access model (Duffy et al., 1988), which suggests that in the case of biased ambiguous words, the preceding biasing context boosts the activation of the less frequent meaning so that it is accessed at or near the same time as the dominant interpretation. The resulting inflated processing times on these words are due to a time-consuming selection process between the meanings. Contrary to the selective access accounts, this model suggests that access of lexically ambiguous words is exhaustive, but not autonomous, in that factors such as relative meaning frequency and context influence the order in which the words are accessed.

\section{REFERENCES}

BindeR, K. S., \& MORRIS, R. K. (1995). Eye movements and lexical ambiguity resolution: Effects of prior encounter and discourse topic. Journal of Experimental Psychology: Learning, Memory, \& Cognition, 21, 1-11.

DOPKINS, S., MorRIs, R. K., \& Rayner, K. (1992). Lexical ambiguity and eye fixations in reading: A test of competing models of lexical ambiguity resolution. Journal of Memory \& Language, 31, 461-477.

DufFy, S. A., MORRIS, R. K., \& RaYNER, K. (1988). Lexical ambiguity and fixation times in reading. Journal of Memory \& Language, 27, 429-446.

FORSTER, K. I. (1979). Levels of processing and the structures of the language processor. In W. E. Cooper \& E. C. T. Walker (Eds.), Sen- 
tence processing: Psycholinguistic studies presented to Merrill Garrett (pp. 27-85). Hillsdale, NJ: Erlbaum.

FRANCIS, W., \& KUČERA, H. (1982). Word frequency counts of modern English. Providence, RI: Brown University Press.

Frazier, L., \& RaYNER, K. (1990). Taking on semantic commitments: Processing multiple meanings vs. multiple senses. Journal of Memory \& Language, 29, 181-200.

Kellas, G., Martin, C., Yehling, K., Herman, R., \& Vu, H. (1995, November). Contextual strength as a determinant of the subordinate bias effect. Poster presented at the annual meeting of the Psychonomic Society, Los Angles.

Kellas, G., Paul, S. T., Martin, M., \& Simpson, G. B. (1991). Contextual feature activation and meaning access. In G. B. Simpson (Ed.), Understanding word and sentence (pp. 47-71). Amsterdam: Elsevier.

Kellas, G., Vu, H., Martin, C., \& Herman, R. (1994, November). Discourse-level contributions to on-line lexical ambiguity resolution. Poster presented at the annual meeting of the Psychonomic Society, St. Louis.

MarsLEN-Wilson, W. (1975). Sentence perception as an interactive, parallel process. Science, 189, 226-228.

MORRISON, R. E. (1984). Manipulations of stimulus onset delay in reading: Evidence for parallel programming of saccades. Journal of Experimental Psychology: Human Perception \& Performance, 10, 667-682.

NeILl, W. T. (1989). Lexical ambiguity and context: An activationsuppression model. In D. S. Gorfein (Ed.), Resolving semantic ambiguity (pp. 63-83). New York: Springer-Verlag.

NeIll, W. T., Hilliard, D. V., \& CoOper, E. (1988). The detection of lexical ambiguity: Evidence for context sensitive parallel access. Journal of Memory \& Language, 27, 279-287.

Nelson, D. L., McEvoy, C. L., Walling, J. R., \& Wheeler, J. W., JR. (1980). The University of South Florida homograph norms. Behavior Research Methods \& Instrumentation, 12, 16-37.

Paul, S. T., Kellas, G., Martin, M., \& Clark, M. B. (1992). The influence of contextual features on the activation of ambiguous word meanings. Journal of Experimental Psychology: Learning, Memory, \& Cognition, 18, 703-717.

RAYNER, K., \& DUFFY, S. A. (1986). Lexical complexity and fixation times in reading: Effects of word frequency, verb complexity, and lexical ambiguity. Memory \& Cognition, 14, 191-201.

RaYNER, K., \& Frazier, L. (1989). Selection mechanisms in reading lexically ambiguous words. Journal of Experimental Psychology: Learning, Memory, \& Cognition, 15, 779-790.

RaYNer, K., PACHT, J. M., \& DUFFY, S. A. (1994). Effects of prior encounter and discourse bias on the processing of lexically ambiguous words: Evidence from eye fixations. Journal of Memory \& Language, 33, 527-544.

RAYNER, K., \& POLLATSEK, A. (1989). The psychology of reading. Englewood Cliffs, NJ: Prentice-Hall.

SERENo, S. C. (1995). The resolution of lexical ambiguity: Evidence from an eye movement paradigm. Journal of Experimental Psychology: Learning, Memory, \& Cognition, 21, 582-595.

Sereno, S. C., Pacht, J. M., \& RaYner, K. (1992). The effect of mean- ing frequency on processing lexically ambiguous words: Evidence from eye fixations. Psychological Science, 3, 296-300.

SiMPSON, G. B. (1984). Lexical ambiguity and its role in models of word recognition. Psychological Bulletin, 96, 120-136.

Twilley, L. C., Dixon, P., TAYloR, D., \& ClaRK, K. (1994). University of Alberta norms of relative frequency for 566 homographs. Memory \& Cognition, 22, 111-126.

\section{NOTES}

1. The original Kellas et al. (1995) materials consisted of 56 ambiguous words. However, according to local norms, 14 of those items were balanced ambiguous words, so they were removed from the materials. An additional 10 items were removed for other reasons (see text for details)

2. Strength of context was established in a norming task conducted by Kellas et al. (1995). Subjects rated the strength of the context preceding the target word on a scale from 1 to $5(1=$ weakly biasing; $5=$ strongly biasing). For their entire set of materials, the average strength rating for the strongly biasing context was 3.7 , and the average strength rating for the weakly biasing context was 1.99 . The strength ratings were averaged across dominant and subordinate meanings. For the set of materials that we used, the average strength rating for the strongly biasing contexts was 3.8 , and the average strength rating for the weakly biasing contexts was 1.99 . There was no overlap in the distributions for the two context types. It should also be noted that we collected norming data from 24 University of Massachusetts subjects and obtained similar ratings.

3. Another measure of processing time that is often reported is the first-fixation duration, which is the duration of the first fixation on a target word independent of the number of fixations that are made on the word before moving to another word. As with prior studies of lexical ambiguity using eye movement measures, the pattern of the first-fixation duration data was the same as that of the gaze duration data.

4. Kellas et al. (1995) used the norms of Nelson, McEvoy, Walling, and Wheeler (1980) to determine whether an ambiguous word was biased or balanced; $80 \%$ of the items in their sample were from the Nelson et al. norms (personal communication). For the 14 words that we excluded because they were balanced words according to our local norms and the Twilley norms, there is clearly a discrepancy with the Nelson norms. The average bias for these words according to the Nelson norms was .79 , but according to the Twilley norms it was .61, and according to our local norms, it was .58. Clearly, the local norms and the Twilley norms are quite consistent, while the Nelson norms are different. Regional differences apparently are significant factors when using norms of these types. It should be noted that we collected our local norms by having subjects evaluate a list of words (both ambiguous and unambiguous). The subjects were instructed to provide the first word that came to mind and then to use the original word in a sentence.

(Manuscript received September 12, 1996; revision accepted for publication October 15, 1997.) 